\title{
Le front de chevauchement au Jebel Bou El Hanèche- Kalâat Khasba (Tunisie centro-septentrionale). Mise en évidence à partir des donnés géologiques et géophysiques
}

\section{The thrust front in the Jebel Bou El Hanèche - Kalâat Khasba (Central-northern Tunisia). Integration of geological and geophysical data}

\author{
I. Ezzine ${ }^{1}$, M. Jaffal ${ }^{2}$, M. Ben Youssef ${ }^{3}$, F. Zargouni $^{1}$, M. Ghanmi ${ }^{1}$
}

RESUME

En Tunisie Centro-Septentrionale, la bordure septentrionale du fossé de Kalâat Khasba a été considéré comme une bordure d'effondrement à jeu normal, ayant fonctionnée du Crétacé inférieur jusqu'au Quaternaire. Une étude de terrain et l'interprétation de profils de sismique réflexion permettent de proposer un nouveau modèle tectonique pour ce fossé et du massif adjacent de Bou El Hanèche.

La bordure septentrionale du bassin de Kalâat Khasba a été réactivée à l'Eocène en faille inverse. Cette bordure a une valeur de front de chevauchement sur lequel se met en place le pli de propagation de Bou El Hanèche par rapport à l'avant pays de la chaîne alpine.

La compression Eocène entraîne la mise en place du pli de Bou El Hanèche à la faveur d'un niveau de décollement situé dans les séries triasiques. Cette déformation est contrôlée par un réseau de failles normales héritées de la phase distensive présumée aptienne. La dynamique compressive continue depuis l'Eocène engendrant la propagation de ce pli. Lorsque la rampe atteint les calcaires de la formation Abiod, le pli se bloque. Ce blocage entraîne la rupture du dressant et la création, vers le Nord du pli, d'un deuxième chevauchement, ce qui implique une évolution du système de chevauchement dans une séquence inverse ou «out of sequence».

Mots clés: Tunisie Centro-Septentrionale, Kalâat Khasba, chevauchement, pli de propagation de rampe, séquence inverse, sismique réflexion.

\begin{abstract}
In Central-northern Tunisia, the septentrional border of Kalâat Khasba trough was considered as a normal fault which has functioned since the Cretaceous to Quaternary periods. A stratigraphic and geophysical recent study of the Kalâat Khasba trough and the Jebel Bou El Hanèche fold area, allows us to propose a new tectonic model. The Northern border was reactivated as reverse fault during the Eocene and was considered as a thrust front. A compressive Eocene event resulted in formation of Bou El Hanèche fold (fault propagation fold) and in determination of a decollement level situated in the Triassic series. This deformation was controlled by the reactivation of normal faults inherited from a distensive aptien phase.

The compressive deformation was amplified by the propagation of this fold and, when a ramp reached the competent carbonates series of late Campanian-lower Maâstrichtien times, the fold was stacked, involving a breakthrough steep limb and creating a second thrust on the North. The order of evolution of these events shows an out of sequence thrust.
\end{abstract}

Keywords: Central-northern Tunisia, Kalâat Khasba, thrust, fold propagation fault, out of sequence thrust, seismic reflection.

\footnotetext{
1 Unité de Géologie structurale et appliquée, Faculté des Sciences de Tunis, Université Tunis-El Manar, Campus Universitaire, 1060, Tunis, Tunisie.Email: ezzine.i@gmail.com, fouadzargouni@gmail.com, ghanmi.mohamed@gmail.com

2 Laboratoire de Géo-Ressources, Département des Sciences de la Terre, Faculté des Sciences et des Techniques, Université Cadi Ayyad, Marrakech, Maroc. Email: jaffal@fstg-marrakech.ac.ma

3 C.E.R.T.E Centre des Recherches, des Technologies des Eaux de Borj Cédria, B.P, 273; Soliman 8020, Tunisie.

Email: benyoussefhim@yahoo.fr
} 


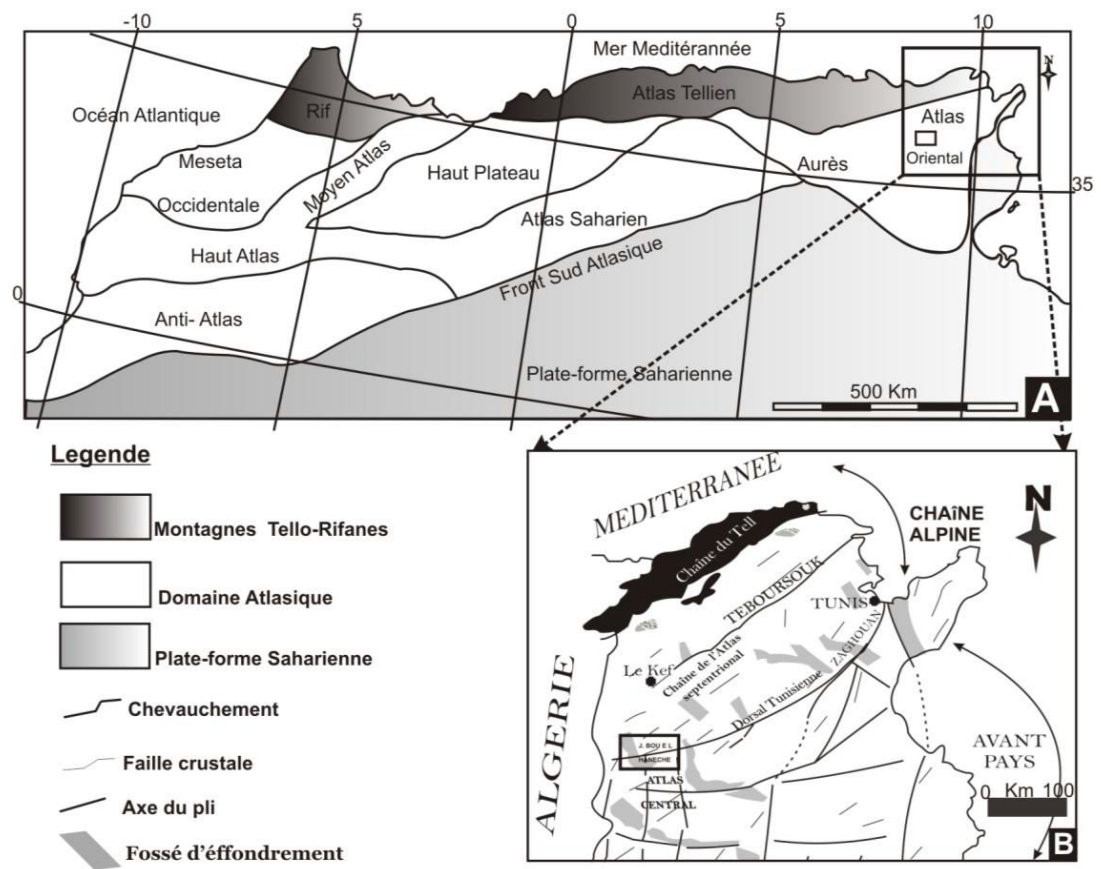

Figure 1.-A: Carte géologique générale de l'Afrique du Nord avec les principaux domaines structuraux. B: Carte structurale de la Tunisie avec localisation de la zone d'étude (Ghanmi, 2003).

\section{Introduction}

En Tunisie Centro-Septentrionale, l'accident bordant la partie sud du Jebel Bou El Hanèche a été considéré comme une bordure d'effondrement à jeu normal, ayant fonctionnée du Crétacé inférieur jusqu'au Quaternaire, Caire (1977); Ben Ayed (1986, 1993); Chihi (1995, 2003). Cet accident présente une direction allant de $\mathrm{N} 80^{\circ}$ à $110^{\circ}$, rejouant en dextre à composante inverse. Cette dernière a la valeur d'un front de chevauchement sur lequel se met en place le pli du Jebel Bou El Hanèche, Ezzine (2007). La révision de cette région fondée sur l'intégration des données géologiques et géophysiques (profils sismiques), montre qu'il s'agit d'une structure anticlinale de direction ENE-WSW, Ezzine (2007).

Cet article présente les résultats d'une étude du contact entre deux entités géologiques de la Tunisie centro-septentrionale, en l'occurrence, le bassin de Kalâat Khasba et le massif de Jebel Bou El Hanèche. Cette étude est basée sur l'analyse des données géologiques de surface et géophysiques de subsurface (sismique réflexion) enregistré dans le bassin de Kalâat Khasba; apporte de nouvelles précisions sur la nature du contact précité.
A la lumière de l'ensemble des résultats obtenus, et tenant compte de la corrélation entre ces deux types de données, l'évolution cinématique de la structure plissée de Jebel Bou El Hanèche et la géodynamique du bassin de Kalâat Khasba sont discutées.

\section{Contexte géologique}

Lors de la convergence de la plaque africaine et la plaque eurasiatique, la marge nord africaine a été soumise à des contraintes compressives durant le Tertiaire, aboutissant à la genèse des chaines alpines nord africaine ou des Maghrébides, Durand Delga (1980). Cette chaine se prolonge du Maroc jusqu'à la Tunisie, où elle montre quatre unités majeures: *Dans sa partie occidentale (au Maroc): l'AntiAtlas, le Haut Atlas, le Moyen Atlas et le Rif. * En Algérie Occidentale, elle est constituée par deux chaînes importantes: l'Atlas Tellien et l'Atlas Saharien séparé par les Haut Plateaux, enfin, vers l'Est de l'Algérie ces deux chaînes fusionnent pour donner une chaîne unique qui constitue en Algérie orientale et en Tunisie l'Atlas Oriental ou l'Atlas Tunisien (Fig.1). Ce dernier est subdivisé en Atlas 


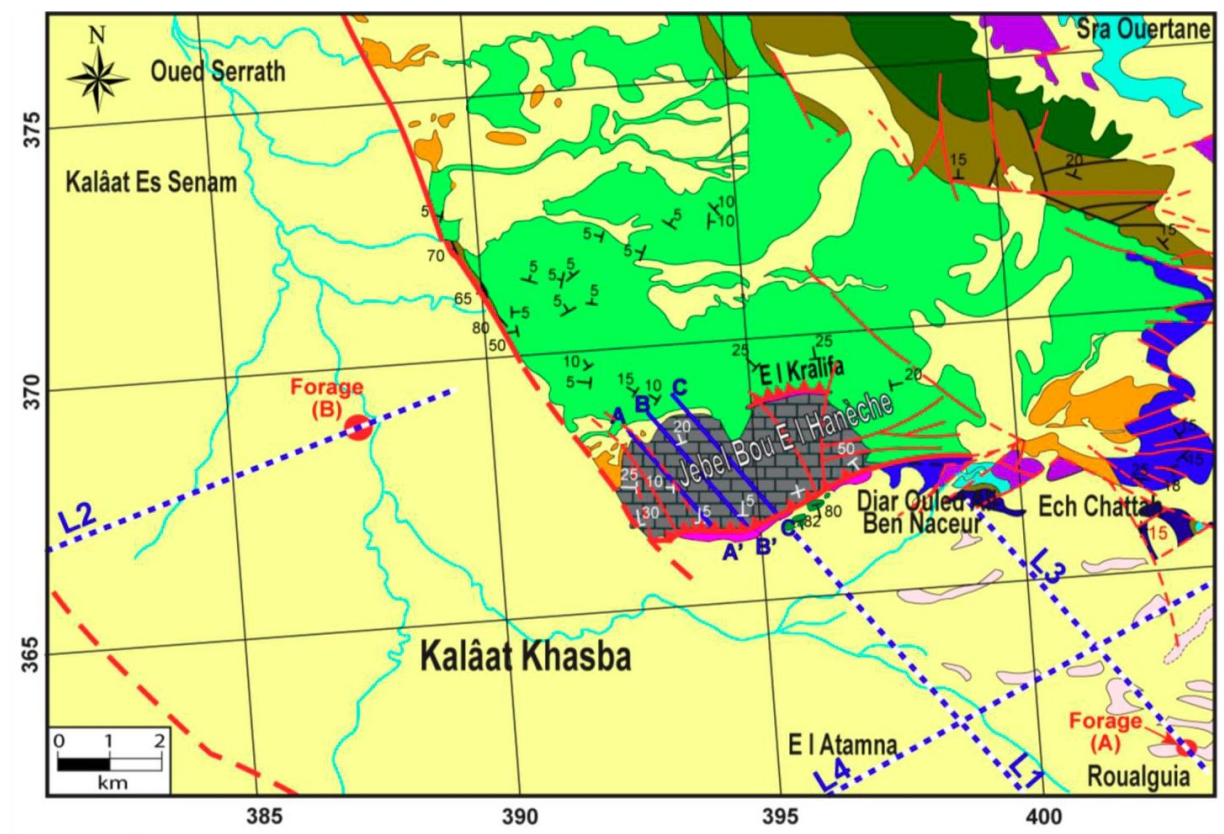

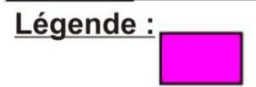

(1)

(2)

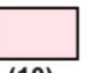

(10)

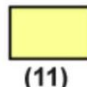

(11)

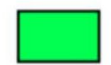

(3)

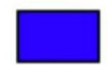

(4)

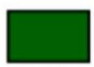

(5)

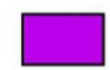

(6)

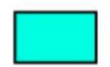

(7)

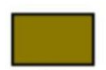

(8)

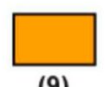

(9)

Figure 2.-Carte géologique simplifiée du secteur étudié, avec localisation des coupes et des lignes sismiques étudiées (d'après Lehotsky et Bujonowsky, 1978; modifiée par Ezzine, 2007). (1) Trias évaporitiques; (2) Aptien (Fm. SERJ, marnes bioclastiques à gastéropodes, lamellibranche et échinodermes avec d'importants niveaux de calcaires et de dolomies riches en orbitolines); (3) Albo-Cénomanien (Fm. FAHDENE, alternance des niveaux argileux avec des calcaires en plaquettes); (4) Turonien inférieur à moyen (FM. ANNABA et BERINO, marnes incluant des niveaux calcaires); (5) Campanien supérieur Mâastrichtien inférieur (Fm. ABIOD, deux massives barres de calcaires séparées par une alternance marno-calcaires); (6) Mâastrichtien supérieur - Paléocène (Fm. HARIA, alternance de marnes et d'argiles grises); (7) Paléocène terminal - Yprésien basal (Fm. Chouabine, séries phosphatées); (8) Yprésien - Lutétien p.p. (Fm. GARIA, calcaires massifs à nummulites); (9) Lutétien - Priabonien (Fm. SOUAR, marnes jaunes à débris de bivalves); (10) Quaternaire; (12) Réseau hydrographique; (13) Chevauchement; (14) Failles; (15) Pendages; (16) Emplacement des profils sismiques; (17) Emplacement des coupes géologiques.

Septentrional, centrale et méridionale. L'Atlas Central est caractérisé par des plis de direction Atlasique NE-SW souvent associées à des fossés d'effondrement de direction NW-SE. En effet, le massif de Bou El Hanèche fait partie de l'Atlas Tunisien centro-septentrionale à fossés d'effondrements. La position géologique de ce massif associé au fossé de Kalâat Khasba, a attiré plusieurs auteurs, Pervinquière (1903); Castany (1948, 1951); Dubourdieu (1956); Burollet (1957); Jauzein (1967); Richert (1971); Caire (1977); Ben Ayed \& Kessibi (1985); Ben Ayed 1(986, 1993); Chihi (1995) et révèle plusieurs hypothèse concernant la chronologie de sa mise en place et l'ouverture du fossé de Klâats Khasba.

\section{Analyse des nouvelles données géologiques}

Le massif de Bou El Hanèche se localise sur le croissement des failles de direction NW-SE et ENEWSW qui bordent le fossé de Kalâat Khasba au NE. Il est limité au Sud par la plaine d'El Atamna, au Nord par le synclinal de Sra Ouertane, à l'Ouest par la plaine de Kalâat Es Senam et au Sud-Est par le synclinal de Roualguia (Fig. 2). Dans le secteur d'étude les séries lithostratigraphiques s'étalent du Trias jusqu'au Quaternaire et présentent des discontinuités concernant les séries du Jurassique, du Crétacé inférieur, du Miocène inférieur et de l'Oligocène (Fig. 3). 


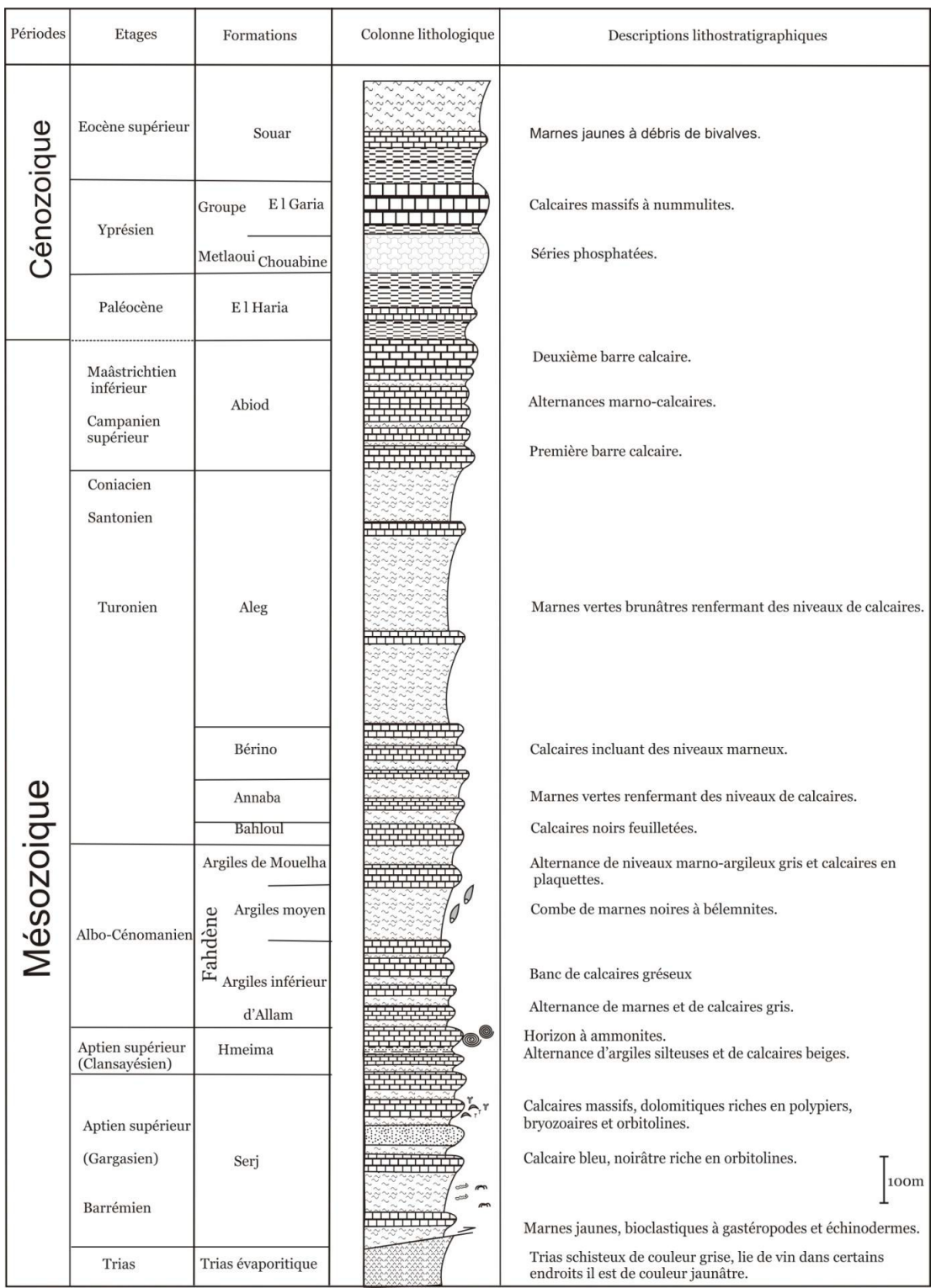

Figure 3.-Colonne lithostratigraphique synthétique du secteur d'étude.

Le massif de Bou El Hanèche se présente sous forme d'une structure anticlinale plissée de direction ENE-WSW dont le cœur est occupé par la formation Serj d'âge Barrémo-Aptien. Il est affecté par trois familles de failles de direction NW-SE, NNWSSE à N-S et ENE-WSW à E-W, Ezzine (2007) (Fig. 2).

Le massif de Bou El Hanèche est caractérisé par deux flancs de pendage et de lithologie différentes séparés par un accident principal E-W ayant valeur de rampe (Fig. 4 et 5): Le flanc Nord (amont) long représenté par les séries aptiennes à faible pendage $\left(15^{\circ}\right.$ à $\left.20^{\circ}\right)$ et le flanc sud (aval) court représenté par les séries du Crétacé terminal à fort pendage (80 environ).

La limite méridionale de ce massif est matérialisée par une faille de direction $\mathrm{N} 75^{\circ}$ à fort pendage $\left(70^{\circ}\right.$ vers le NW) qui le sépare du bassin de Kalâat Khasba. L'analyse du miroir de cette faille et l'interprétation des tectoglyphes signalent la présence 

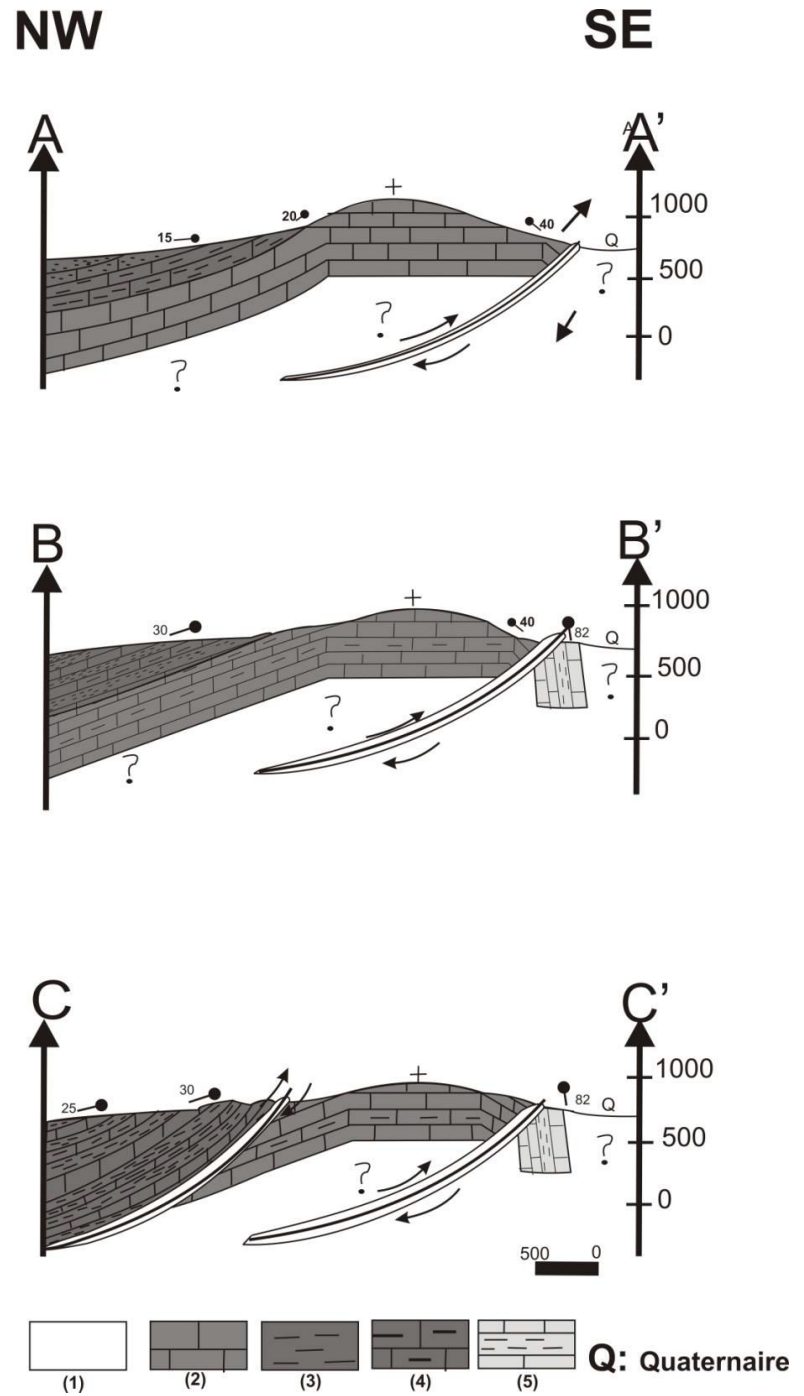

Figure 4.-Coupes géologiques sériées illustrant l'évolution latérale de la structure plissée de Jebel Bou El Hanèche. (1) Trias évaporitique; (2) Calcaire de la formation Serj (BarrémoAptien); (3) Séries argilo-calcaires de la formation Fahdène (Albo-Cénomanien); (4) Séries argileuse de la formation Fahdène (Albo-cénomanien) (5) Séries calcaires (Campanien supérieurMâastrichtien inférieur).

de deux générations de stries, les premières horizontales et les secondes verticales, Ezzine (2007). La coexistence de ces deux générations sur le même plan de faille traduit un polyphasage; un jeu horizontal en décrochement pur et un jeu vertical. Il en résulte que cet accident aurait fonctionnée en un décrochement dextre.

Les observations géologiques récentes effectuées sur cet accident, nous ont permis de découvrir du matériel triasique qui le jalonne d'une manière lenticulaire. A Ouled Ali Ben Naceur, ce matériel, de

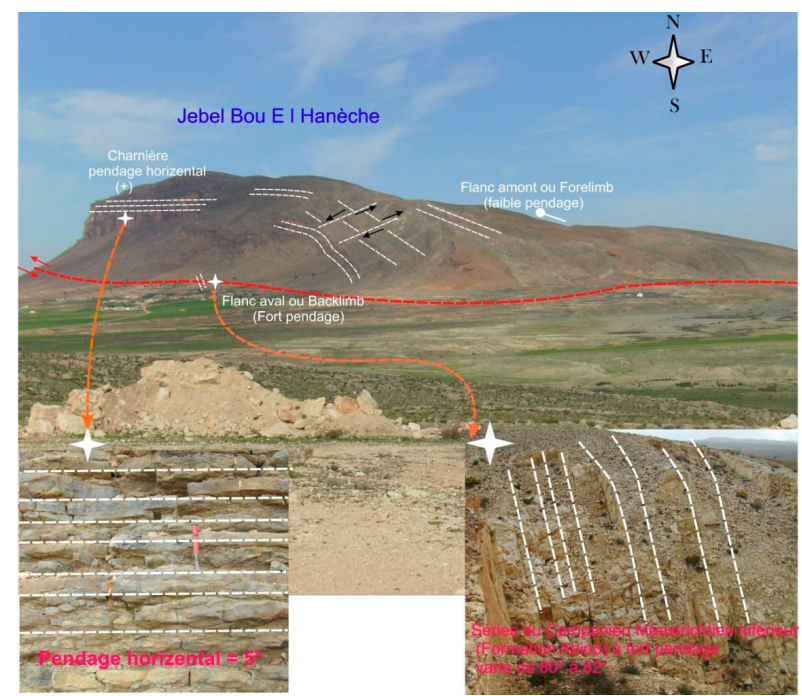

Figure 5.-Vue panoramique du massif de Bou El Hanèche.

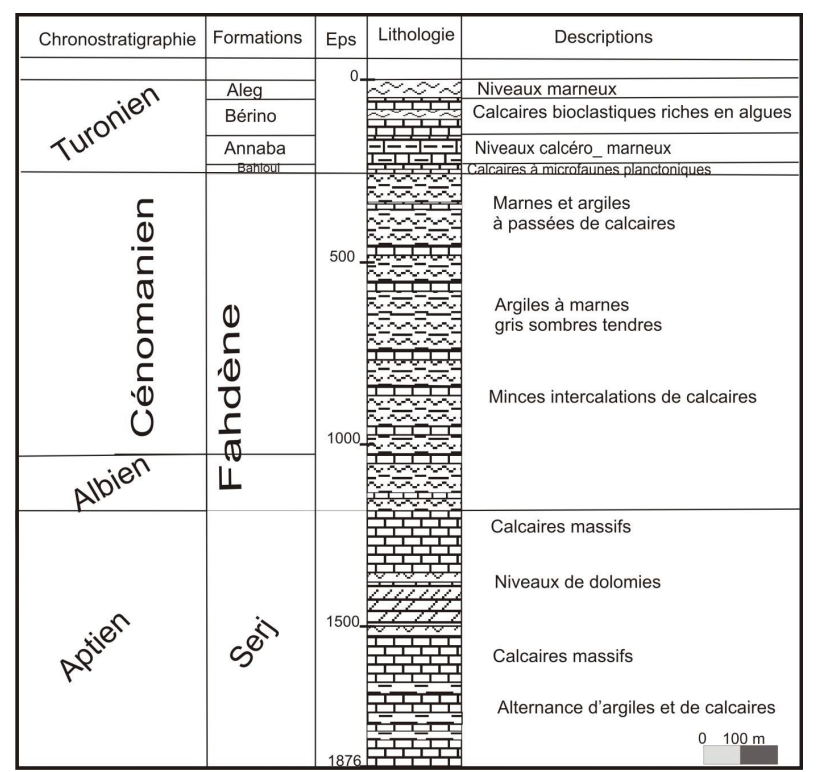

Figure 6.-Colonne lithostratigraphique du puits $(A)$

nature chaotique et de couleur lie de vin, met en contact des séries aptiennes contre celles du Crétacé supérieur. Dans la zone d'El Kralifa, le matériel triasique de même lithologie, jalonne l'accident $\mathrm{N}$ $90^{\circ}$ d'El Kralifa et assure le contact entre les séries de l'aptien inférieur et les séries albiennes (Fig. 2).

Une corrélation des coupes sériées de direction NW-SE relevées à travers le massif de Bou El Hanèche révèle l'existence d'une importante structure dissymétrique formée par l'association d'un pli et d'une faille inverse (Fig. 4). 


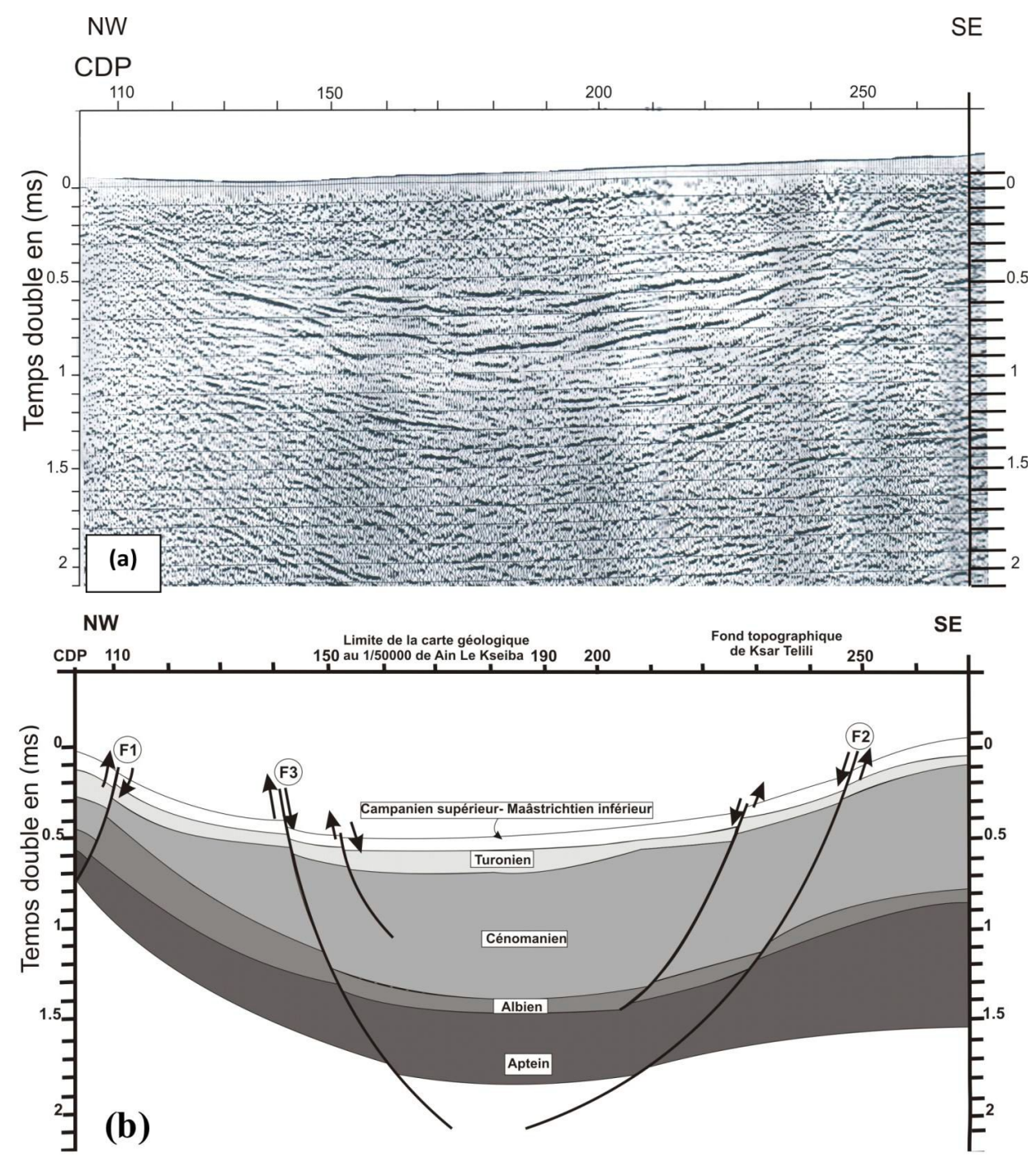

Figure 7.-Section sismique L1; (a) Non interprétée, (b) Interprétée.

\section{Analyse des données sismiques}

Dans le cadre de la recherche pétrolière, le bassin de Kalâat Khasba a fait l'objet de nombreuses campagnes de prospection géophysique et d'exploration par forages profonds, ce qui a permis d'enregistrer plusieurs profils de sismique réflexion. L'analyse de quelques uns de ces profils a permis de déterminer la structure profonde du bassin de Kalâat Khasba. Les deux profils L1 et L2 sont interprétés après leur calage avec les données des forages A et B (Fig. 2).

Lors de cette étude, nous avons utilisé des lignes sismiques disponibles de l'Entreprise Tunisienne des Activités Pétrolière (E.T.A.P.).

\section{Profil L1}

Le profil sismique L1 a été acquis par la Compagne Générale de Géophysique (CGG), en Mai 1982. La source utilisée est la dynamite. Ce profil présente une échelle horizontale de $1 \mathrm{~mm}$ CDP 35 m 1/25000; une échelle verticale de 3.75 inches par seconde et d'une vélocité de $1 \mathrm{Km} / \mathrm{s}$. Ce profil a été implanté dans le bassin de Kalâat Khasba perpendiculairement à l'axe du bassin et à celui du massif de Bou El Hanèche (Fig. 2). En l'absence de sondage au voisinage immédiat de ce profil, le calage a été effectué à partir du forage (A) (Fig. 2 et 6) par corrélation avec les lignes sismiques L3 et 


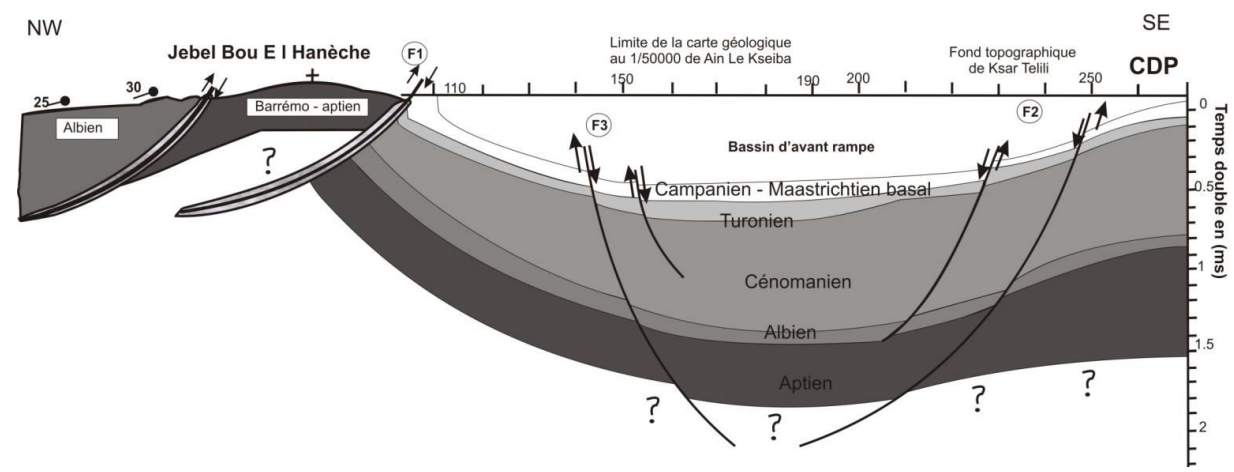

Figure 8.-Coupe interprétative à travers le massif de Bou El Hanèche et le bassin de Kalâat Khasba. D'après le profil sismique L1 et la coupe géologique CC'.

\begin{tabular}{|c|c|c|c|c|}
\hline Chronostratigraphid & Formations & Eps & Lithologie & Descriptions \\
\hline$e^{0^{0}}$ & Segui & 200 & 30 & $\begin{array}{l}\text { Sables, argiles rouges } \\
\text { et conglomérats. }\end{array}$ \\
\hline $\begin{array}{l}\text { Miocène } \\
\text { supérieur }\end{array}$ & $\begin{array}{l}\text { O. Bel } \\
\text { Khedim }\end{array}$ & & 3 & Gypses, argiles et sables \\
\hline $\begin{array}{l}\text { Miocène } \\
\text { moyen }\end{array}$ & Dhouil & $\begin{array}{r}600 \\
800- \\
1000 \\
1200\end{array}$ & 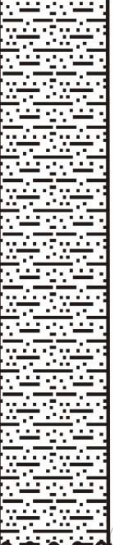 & Argiles sableuses \\
\hline Eocène moyen & Cherahil A & & & Alternance de dolomies, marnes et argiles \\
\hline Miocène moyen & Oum Dhouil & & & Argiles sableuses \\
\hline $\begin{array}{l}\text { Eocène } \\
\text { inférieur }\end{array}$ & $\begin{array}{l}\text { Groupe } \\
\text { Metlaoui }\end{array}$ & 1400 & & $\begin{array}{l}\text { Alternance de dolomies et du marnes } \\
\text { Séries phosphatées }\end{array}$ \\
\hline $\begin{array}{l}\text { Paléocène } \\
\text { inférieur }\end{array}$ & $\begin{array}{ll}\text { ET } \\
\text { Haria }\end{array}$ & & & Niveaux marneux \\
\hline $\begin{array}{l}\text { Eocene } \\
\text { inférieur }\end{array}$ & $\begin{array}{l}\text { Groupe } \\
\text { Metlaoui }\end{array}$ & & & $\begin{array}{l}\text { Alternance de dolomies et du marnes } \\
\text { Niveaux phosphatees }\end{array}$ \\
\hline $\begin{array}{l}\text { Eocène } \\
\text { inférieur }\end{array}$ & $\begin{array}{l}\text { Groupe } \\
\text { Metlaoui } \\
\end{array}$ & 1600 & & $\begin{array}{l}\text { Alternance de dolomies et du marnes } \\
\text { Séries phosphatées }\end{array}$ \\
\hline $\begin{array}{l}\text { Paléocène } \\
\text { inférieur }\end{array}$ & $\begin{array}{l}\text { E I } \\
\text { Haria }\end{array}$ & & & Niveaux marneux \\
\hline $\begin{array}{l}\text { Campanien Sup } \\
\text { Maastrichtien in }\end{array}$ & Abiod & & 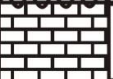 & Calcaires blancs \\
\hline \begin{tabular}{|l|} 
Turonien sup \\
Santonien sup \\
\end{tabular} & Aleg & & & Marnes renfermant des niveaux de calcaires \\
\hline Trias & Trias & 2000 & & Gypses et anhydrites \\
\hline
\end{tabular}

Figure 9.-Colonne lithostratigraphique du puits (B). 

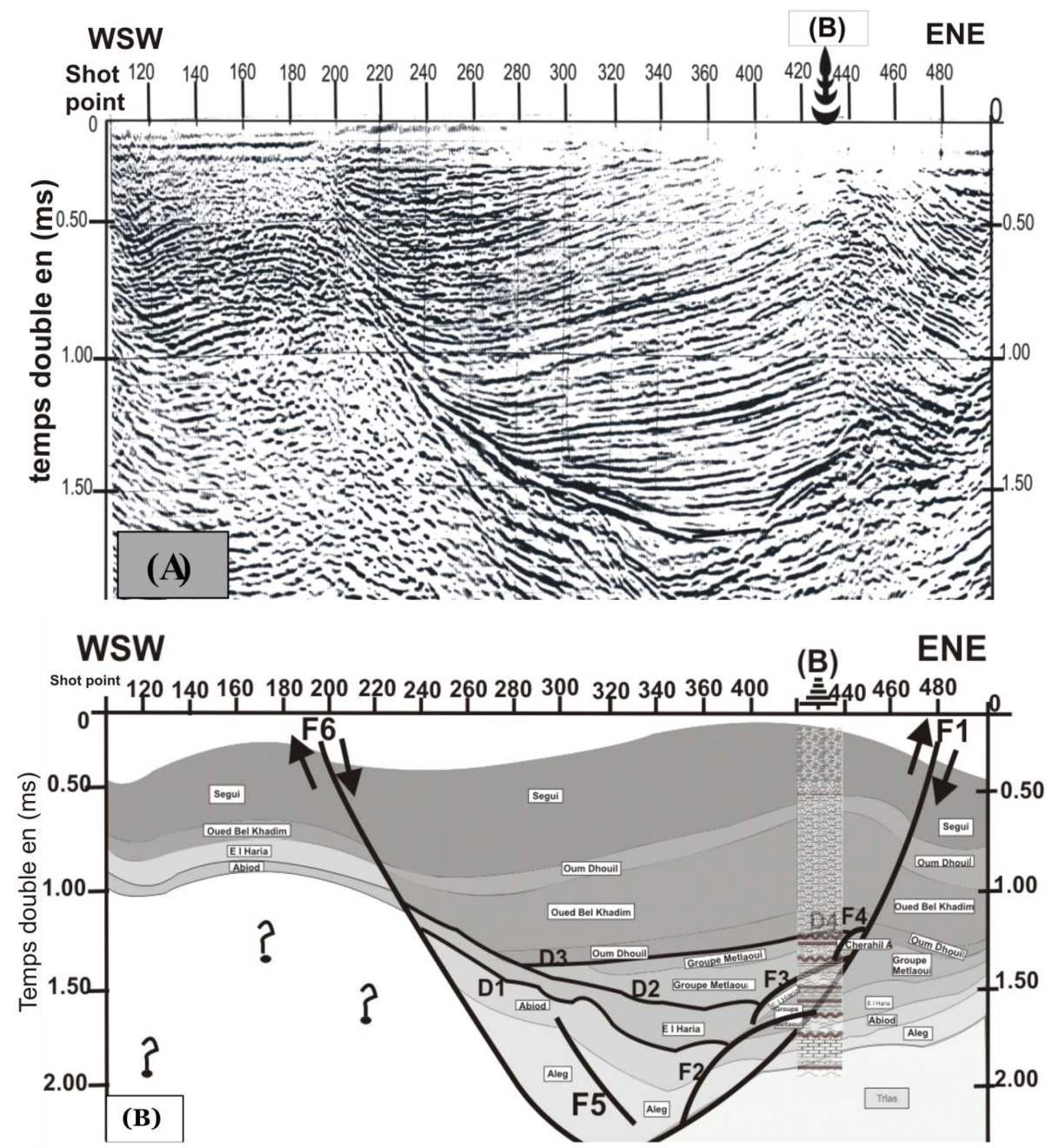

Figure 10.—Section sismique L2; (A) Non interprétée, (B) Interprétée.

L4 (Fig. 2). La convection du temps en profondeur est réalisée en se basant sur une courbe temps-profondeur. L'interprétation de ce profil sismique L1 était trés difficile car il est non migré. L'allure générale de la structure distinguée dans ce profil, montre une gouttière de forme relativement symétrique, à géométrie synclinale limitée de part et d'autre par deux majeures failles normales antithétiques. Ces dernières affectent l'ensemble des séries allant de l'Aptien (Formation Serj) jusqu'aux séries du Campanien supérieur- Maâstrichtien inférieur (Formation Abiod) (Fig. 7a et 7b). Au sein de ce profil on peut signaler la présence d'autres failles normale de moindre importance. Vers la surface, cette gouttière occupe le bassin de Kalâat Khasba.
La prolongation de la ligne L1 avec la coupe géologique $\left(C^{\prime}\right)$ que nous avons réalisée à travers le massif de Bou El Hanèche permet d'avoir une vision plus complété de la structure de la zone d'étude. En effet, la coupe interprétative proposée montre que le bassin de Kalâat Khasba se présente sous forme d'un synclinal d'avant rampe (Fig. 8).

\section{Profil L2}

Le deuxième profil sismique (L2) a été réalisé par MOSBACHER TUNISIA en Mai 1995, à l'Ouest du massif de Bou El Hanèche. Il est orienté parallèlement à la direction d'allongement de ce massif. Son calage a été effectué à l'aide du forage 
pétrolier (B) (Fig. 9) qui le recoupe et en se basant une courbe temps-profondeur. Ce profil présente une échelle horizontale de 42 traces par inches; une échelle verticale de 3.938 inches par seconde et d'une vélocité de $1 \mathrm{Km} / \mathrm{s}$. La séquence de traitement est assurée en adoptant une vitesse de remplacement de $2000 \mathrm{M} / \mathrm{S}$ et une vitesse de réfraction de $1250 \mathrm{M} / \mathrm{S}$ Overbruden vilocity de $1000 \mathrm{M} / \mathrm{S}$.

L'analyse fine de la section migrée de ce profil permet d'identifier quatre discordances angulaires et quatre failles. On distingue de bas en haut (Fig. $10 \mathrm{~B})$ :

- La faille F1:C'est une faille inverse mettant les séries du Turonien supérieur-Santonien supérieur (formation Aleg) en contact direct sur les séries triasiques.

- La discordance D1 entre les calcaires du Campanien supérieur-Maâstrichtien inférieur (formation Abiod) et les argiles du Paléocène inférieur (formation El Haria);

- La discordance D2 qui se situe entre la formation El Haria d'âge Paléocène inférieur et le groupe Metlaoui d'âge Eocène inférieur;

- La faille F2: elle permet le redoublement des séries éocènes inférieur (groupe Metlaoui).

- La faille F3 est responsable du redoublement des séries Paléocène inférieur et Eocène moyen (formation El Haria et le groupe Metlaoui).

- La discordance D3 qui marque le passage du groupe Metlaoui (Eocène inférieur) à la Formation Oum Dhouil (Miocène moyen);

- La discordance D4, qui matérialise la limite entre les alternances dolomitiques, marneuses de la formation Chérahil (A) (Eocène moyen) et la formation Oum Dhouil (Miocène moyen).

Le profil L2 permet également de mettre en évidence plusieurs failles notées F1 à F6 sur la (Fig. 10). Les plus importantes, F1 et F6, ont tendance à se raccorder bien qu'elles présentent des jeux et des pendages opposés. Les failles mises en évidence peuvent être subdivisées en deux familles (Fig. 10a et $10 b)$.

Les dédoublements des séries associés à la formation des duplex permettent de dessiner sur la section sismique des structures imbriquées marquant clairement la signature de la compression Eocène qui se poursuit jusqu'au Pliocène. Ceci est souligné par le jeu inverse de la faille F1 qui affecte les séries pliocène de la formation Segui (Fig. 10a et $10 \mathrm{~b}$ ) et attesté par les études régionales montrant des discordances, Lehotsky \& Bujonovsky (1978).

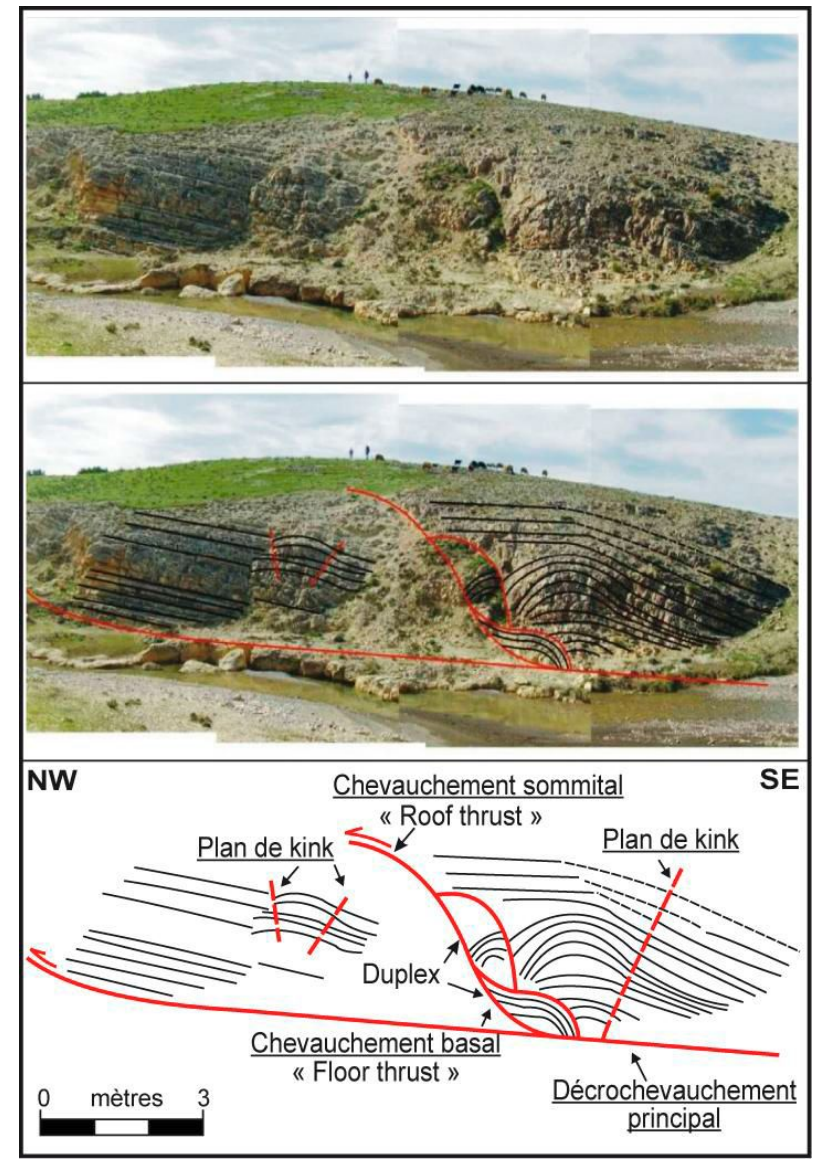

Figure 11.-(a) Evolution cinématique du massif de Bou El Hanèche (1) Trias, (2) Aptien, (3) Albo-Cénomanien, (4) Turonien - Santonien, (5) Campanien - Maâstrichtien. (b) Schéma synthétique illustrant la géodynamique du secteur d'étude de l'AptoAlbien jusqu'à l'actuel.

Le deuxième ensemble est formé par les failles F5 et F6 qui présentent un jeu normal.

\section{Discussion}

Le pli de Bou El Hanèche, situé sur la bordure septentrionale du fossé d'effondrement de Kalâat Khasba, a été considéré dans les anciens travaux de Ben Ayed (1986,1993); Burollet (1956); Caire (1977); Castany (1948, 1951, 1953); Chihi (1995, 2003); Richert (1971) comme une structure monoclinale de direction atlasique (NE-SW). (Fig.2)

L'étude structurale détaillée de cette zone et l'interprétation des profils de sismique réflexion, montrent que ce massif correspond à une structure anticlinale de direction ENE-WSW. L'étude de la variation de pendage montre qu'il s'agirait d'un pli de 


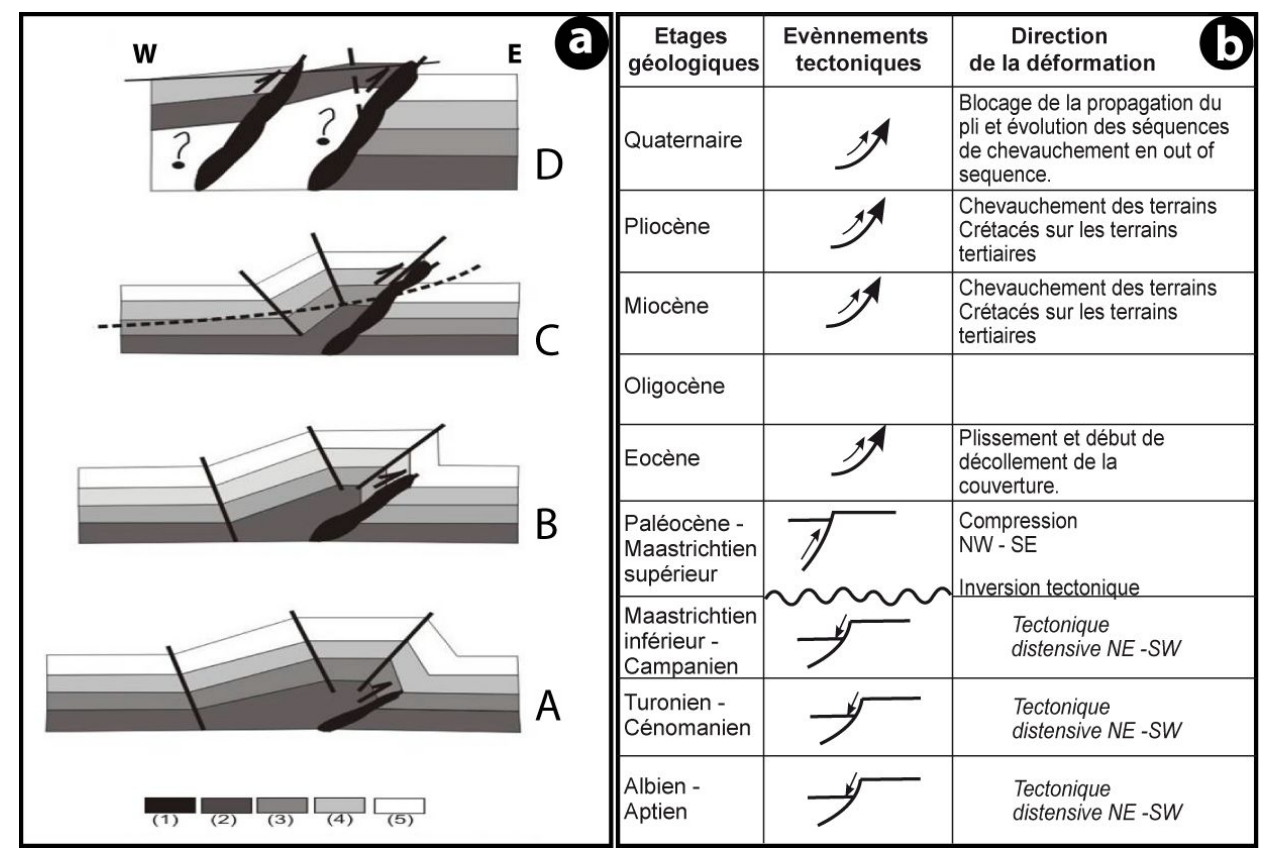

Figure 12.-Vue d'ensemble de la formation calcaire d'Abiod au niveau de l'Oued Bou Gleda.

propagation de rampe utilisant le matériel triasique comme un niveau de décollement, Mercier (1992a, 1992b); Mercier et al. (1992, 1995, 1997).

Les premières pulsations compressives affectant le secteur étudié sont d'âge post Maâstrichtien inférieur. La signature de ces pulsations est enregistrée dans l'Oued Bou Gelada par la présence de structures en «duplex», Merle (1994), au sein de la barre supérieure de la formation Abiod. (Fig.12).

Considérant l'ensemble des résultats obtenus au cours de la présente étude, nous proposons un modèle cinématique qui résume l'évolution de cette zone en quatre stades (Fig. 11a et 11b).

- La réactivation de la bordure septentrionale du bassin de Kalâat Khasba en faille inverse. Celle-ci correspond à une rampe se raccordant à un niveau de décollement basal représenté par du matériel salifère. Le paroxysme de la dynamique compressive est d'âge éocène, de direction NW-SE; il est marqué par le redoublement des séries du groupe Metlaoui par la faille F 2 et les discordances D3, D4 qui sont séparée par la faille F 4 (Fig. 10a et 10b).

Cette bordure septentrionale du bassin de Kalâat Khasba correspond à un front de chevauchement par rapport au domaine d'avant pays de la chaîne alpine, sur lequel se met en place un pli de rampe, «le pli de Bou El Hanèche» dans son premier stade d'évolution ou «Simple step Fault-propagation fold», Suppe \& Medwedeff (1990) ou «Self-similar Fault-propagation fold», Erslev \& Mayborn (1997).

- Le second stade marque le dernier épisode de la compression du Miocène supérieur de direction NW-SE, Ben Ayed (1986,1993); Burollet (1956); Caire (1977); Castany (1948, 1951, 1953); Chihi $(1995,2003)$. Au cours de ce stade, la rampe atteint les séries cénomano-turoniennes. La compression miocène accentue la formation et l'élévation du pli de Bou El Hanèche en permettant sa propagation.

- Le troisième stade est marqué par un changement de la direction de la compression qui devient $\mathrm{N}-\mathrm{S}$, sub-perpendiculaire à la direction du pli. La rampe atteint les calcaires massifs de la formation Abiod (séries compétentes), qui entraînent son blocage. A ce stade de l'évolution, se produit; (i) une accentuation de l'amplitude et de l'altitude du pli de Bou El Hanèche et, (ii) un cisaillement simple associé à la rupture du flanc amont selon un plan à fort pendage $\left(80^{\circ} \mathrm{N}\right)$ situé dans les séries Abiod. Cette rupture est définie selon les travaux de Mercier (1992a, 1992b), Mercier et al. (1992); Martin \& Mercier (1995); Merle (1994), comme étant le 


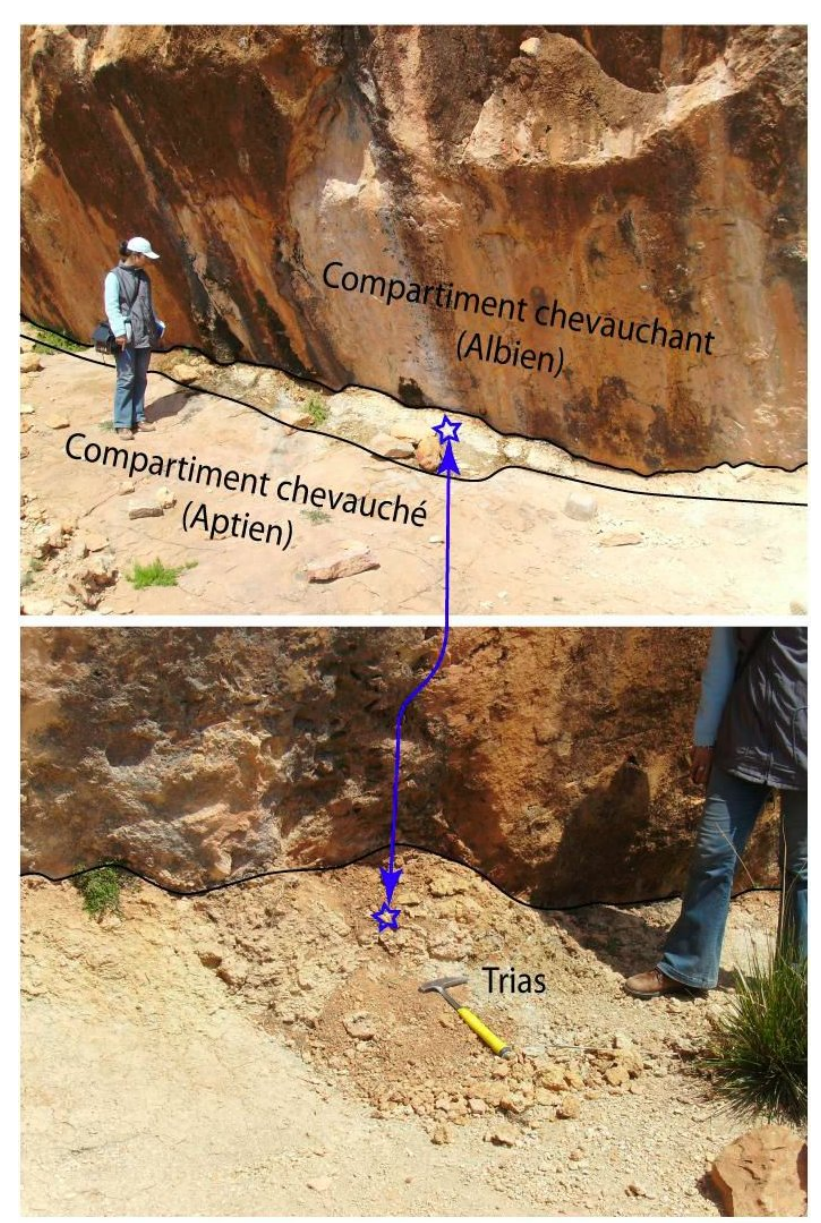

Figure 13.-Chevauchement des séries albiennes sur les séries aptien inférieur.

résultat d'une disposition oblique de la contrainte majeure principale $\sigma 1$ par rapport à la direction générale du pli.

- Le quatrième et le dernier stade correspond à l'évolution post blocage du pli de propagation, Mercier (1992a, 1992b); Mercier \& Mansy (1995); Mercier et al. (1992, 1995,1997). Il est marqué par l'apparition dans la région d'El Kralifa d'un second en arrière de l'accident principal. Ce deuxième chevauchement, de direction $\mathrm{N} 90^{\circ}-65^{\circ} \mathrm{N}$, met en contact les séries albiennes avec les séries de l'aptien inférieur. Le contact est assuré par du matériel triasique. Selon Merle (1994), la création d'un nouveau chevauchement en arrière du premier, traduit une évolution de la propagation du pli selon une séquence inverse ou «out of sequence» (Fig. 11a, 11 b \& Fig. 13). Ce type de déformation est attaqué par l'érosion, qui semble jouer un rôle important dans la configuration actuelle. (Fig. 2 et 5)

\section{Conclusion}

La marge sud de la Téthys en Tunisie, à été soumise à des contraintes compressives, lors de la convergence de la plaque africaine et eurasiatique, aboutissant à la fermeture téthysienne, Dercourt et al. (1978). En Tunisie centrale, cette phase a contribué à l'ouverture de grabens ou fossés d'effondrements de type pull appart de direction NW-SE, Jauzein (1967); Ben Ayed (1986); Chihi (1995) et à la formation de plis atlasiques, Dlala (2002). Dans la partie septentrionale de l'Atlas, les grabens de Bou Ghanem, Kalâat Khasba, Rohia ont une disposition en escalier ou en coude, Ben Ayed (1995 ); Chihi (1995). Au niveau de fossé de Kalâat Khasba, l'intégration des données du terrain et des données sismiques réflexions, montre que le massif du Bou El Hanèche n'est pas un pli simple de direction atlasique situé sur la bordure de ce fossé. Il s'agit en fait d'un pli de propagation de rampe de direction générale ENE-WSW. Ce pli enregistre les effets de plusieurs épisodes compressifs de directions différentes: (Fig.2)

La compression Eocène de direction NW-SE marquée par un redoublement de séries du groupe Metlaoui et les discordances D3 et D4 (Fig. 10a et 10b), elle a permis:

- la réactivation de la faille principale ENEWSW en faille inverse formant la rampe,

- la sollicitation d'un niveau de décollement situé stratigraphiquement au niveau des séries triasiques,

- la mobilisation et la montée à la surface de matériel triasique,

- la formation du pli de Bou El Hanèche dans son stade initial.

Cette compression Eocène est relayée par celle du Miocène supérieur qui a entraîné le développement du pli, puis sa propagation. Cette dernière est assurée par la présence du matériel salifère mis en évidence à l'affleurement pour la première fois dans la région, jouant le rôle de semelle de glissement.

Enfin la compression quaternaire est matérialisée par un changement de la direction de la compression de NNW-SSE à N-S. Dans ce stade la propagation du pli continue (Fig. 8). Lorsque la rampe atteint les séries carbonatées de l'Abiod (Campanien supérieur - Maâstrichtien inférieur), elle engendre un cisaillement et une rupture du dressant selon un plan sub-vertical (pendage $=80^{\circ}$ ). Cette rupture entraîne le blocage de l'évolution du pli et 
la création d'un deuxième chevauchement dans la région d'El Kralifa de direction $\mathrm{N} 90^{\circ}$ en arrière du premier orienté en $\mathrm{N} 75^{\circ}$. Cette disposition des chevauchements montre une évolution selon une séquence inverse de type «out of sequence».

La deuxième faille majeure de direction N 140 à 150 bordant à l'ouest le massif de Bou El Hanèche et le bassin de Kalâat Khasba (Fig. 2), présente un jeu décrochant senestre à pente raide et à regard Ouest, Ben Ayed (1995), ce qui prouve qu'on est en présence d'une rampe latérale, dont la direction du transport est perpendiculaire à la direction du plissement majeur. Ainsi, dans son cadre régional, la bordure septentrionale du bassin de Kalâat Khasba se présente sous forme d'un front de chevauchement par rapport aux bassins d'avant pays de la chaîne alpine, qui forme par conséquent une continuité vers le nord est avec la cicatrice ou le chevauchement de Zaghouan (Fig.1-B). Ce dernier est nommé par la (T2) selon Jauzein (1967). A ce niveau, le front de chevauchement change de direction d'ENE-WSW au niveau du Jebel Bou El Hanèche à NE-SW dans les massifs de ZaghouanRessas, Morgan et al. (1998). Il change donc d'une rampe latérale à une rampe frontale dont la direction du transport est perpendiculaire à la direction générale de raccourcissement (NW-SE). Cette étude permet de mettre en évidence le déplacement du front alpin vers le SW, classiquement les chaines alpines étaient au niveau de front des nappes, Rouvier (1977). En Tunisie Centrale, les plis de propagation de rampe jouent un rôle important dans la localisation des structures pièges de pétrole tel que le cas du Dj. Bou El Hanèche.

\section{Références}

Ben Ayed, N. (1986). Évolution tectonique de l'avantpays de la chaîne alpine de Tunisie du début du Mésozö̈que à l'actuel. Thèse d'Etat, Université de Paris, Sud-centre d'Orsay, 347 pp.

Ben Ayed, N. (1993). Évolution tectonique de l'avant pays de la chaîne alpine de Tunisie du début du Mésozoique à l'actuel. Annuaire des Mines et Géologie, 32: 285.

Ben Ayed, N \& Kessibi, M. (1985). Mise en évidence de deux couloirs de décrochement E-W dextre en bordure de la plateforme saharienne (Tunisie méridionale). Acte 1er Congrès Nationale des Sciences de la Terre (Tunis, 1981), Annuaire des Mines et Géologie, 31: 291-302.

Burollet, P. F. (1956). Contribution à l'étude stratigraphique de la Tunisie Centrale. Annuaire des Mines et Géologie, 18: 1-352.
Caire, A. (1977). Interprétation tectonique unitaire de l'atlas tunisien à fossés. Comptes Rendus de la Académie des Sciences, Paris, 284: 349-352.

Castany, G. (1948). Les fossés d'effondrement de la Tunisie. Géologie et hydrogéologie. 1er fasc. Plaine de Grombalia et cuvettes de Tunisie orientale. Annuaire des Mines et Géologie de Tunisie, 3: 1-126.

Castany, G. (1951). Sur l'âge récent de la phase ultime de diastrophisme majeur de l'Atlas tunisien original. IXXe Congrès A.F.A.S., Tunis, 103-105.

Castany, G. (1953). Le plissement quaternaire en Tunisie. Comptes Rendus Sommaires Société Géologique de France, 11: 198-200

Chihi, L. (1995). Les fossés néogènes à quaternaires de la Tunisie et de la mer pelagienne: Etude structurale et leur signification dans le cadre géodynamique de la Méditerranée centrale. Thèse de Doctorat d'Université, Tunis. El Mannar II. 568 pp.

Chihi, L. (2003). Place de la Tunisie dans l'évolution géodynamique de la Méditerranée occidentale du Néogène à l'actuel. Résumé A.T.E.I.G: La chronologie des événements tectoniques en Tunisie, 43-47.

Dercourt, J.; Zenenshaain, L.P.; Ricou, L.E.; Kazmin, V.G.; Le Pichon, X.; Knipper, A.C.; Grandjaquet, C.; Ssbortchikov, I.M.; Geyssand, J.; Lepunier, C.; Westphal, M.; Bazhenov, M.; Lauer, J.P. \& Biju-Duval, B. (1978). Geological evolution of Tethys from the atlantic to the Pamir since the Lias. Tectonophysics, 123: 241-315. doi:10.1016/0040-1951(86)90199-X

Dlala, M. (2002). La tectonique distensive synsédimentaire d'âge campanien maastrichien en Tunisie: Implication sur l'évolution géodynamique de la marge nord africaine. Comptes Rendus de l'Académie des Sciences, Paris, 334: 135-140.

Dubourdieu, G. (1956). Etude géologique de la région de l'Ouenza (confins algéro-tunisiens). Bulletin du Service de la Carte Géologique de l'Algérie, 10: 1-659.

Durand-Delga, M. (1980). La méditerranée occidentale: étape de sa genèse et problèmes structuraux liés à celle-ci. In: Livre jubilaire de la Société Géologique de France, 1830-1980 (editors?). Mémoire hors série de la Societé Géologique de France, Paris, 10, 203224.

Erslev, E.A \& Mayborn, K. R. (1997). Multiple geometries and modes of fault-propagation, folding in the Canadian thrust belt. Journal of Structural Geology. 19: 321-335. doi:10.1016/S0191-8141(97)83027-1

Ezzine, I. (2007). Apport des données de surface et de subsurface (sismique reflexion) dans l'étude des structures associées aux fossés d'effondrements. Exemple: Le massif de Bou El Hanèche Tunisie centro-septentrionale. Mémoire de Master, Université Tunis El Manar, Faculté des Sciences de Tunis, 90 pp.

Ghanmi, M. (2003). Géodynamique de la plateforme saharienne et sa marge septentrionale au Crétacé, son évolution vers l'Atlas septentrional, interférences du rifting et de l'halocinèse. Habilitation à direction de recherches, Université Tunis El Mannar II, 347 pp.

Jauzein, A. (1967). Contribution à l'étude géologique des confins de la dorsale tunisienne (Tunisie septen- 
trionale). Annuaire des Mines et Géologie de Tunisie, 22: $1-475$.

Lehotsky, I. \& Bujonowsky, A. (1978). Carte géologique à 1/50.000 de Kalat Es Senan. Publications du Service Géologique de la Tunisie.

Martin J. \& Mercier E.(1995). Héritage distensif et structuration chevauchante dans une chaîne de couverture: apport de l'équilibrage par modélisation géométrique dans le Juras nord-occidental. Bulletin de la Société Géologique de France, 167: 101-110.

Mercier, E. (1992a). Les plis de propagation transportés: modélisation et exemple. Habilitation à diriger les recherches, Université Cergy-Pontoise.

Mercier, E. (1992b). Une évolution possible des chevauchements associés aux plis de propagation: le transport sur plat (modélisation et exemple). Bulletin de la Société Géologique de France, 163: 713-720.

Mercier, E. \& Mansy, J.L. (1995). Le blocage du transport sur le plat des plis de propagation: une cause possible des chevauchements hors séquence. Geodynamica Acta, 8: 199-210.

Mercier, E.; Outtani, F.; Frizon De Lamotte, D. \& Ghandriche, H. (1995). Geometry of fault propagation folds: method and application; Comment. Tectonophysics 245, 111-113. doi:10.1016/0040-1951(94)00138-Y

Mercier, E; Outtani, F. \& Frizon De Lamotte, D. (1997). Late stage evolution of fault propagation folds: princi- ples and example. Journal of Structural Geology, 19: 185-193. doi:10.1016/S0191-8141(96)00081-8

Merle, O. (1994). Nappe et chevauchement. Masson, Paris, Milan, Barcelone, 137 pp.

Morgan, M. A.; Grocott, J. \& Moody, R.T.J. (1998). The structural evolution of the Zaghouan-Ressas belt, Northern Tunisia. Geology Society, London, Special Publications, 132: 405-422. doi:10.1144/GSL.SP.1998.132.01.23

Pervinquiere L., (1903). Etude géologique de la Tunisie centrale. Régence de Tunis, Direction génerale des Travaux publics, Carte géologique de la Tunisie, F. de Rudeval, Paris, 360 pp.

Richert, J. P. (1971). Mise en évidence de quatre phases tectoniques successives en Tunisie. Notes du Service Géologique de Tunisie 34, Travaux de Géologie tunisienne, 4: 114-121.

Rouvier, H. (1977). Géologie de l'extrême nord Tunisien: tectoniques et Paléogéographies superposées à l'extrémité Nord-orientale de la chaine maghrébin. Thèse d'état de l'université de Paris VI, France, 703 pp.

Suppe, J. \& Medwedff, D.A. (1990). Geometry and kinematics of fault-propagation folding. Eclogae Geologicae Helvetiae, 83: 409-454.

Recibido el 19 de julio de 2010 Aceptado el 20 de junio de 2011 Publicado online el 12 de julio de 2012 